\title{
MONITOREO DE LA DIGESTIÓN ANAEROBIA DE DESECHOS DEL CENTRO DE FAENAMIENTO DE QUITO
}

\section{MONITORING OF ANAEROBIC WASTE DIGESTION AT QUITO SLAUGHTERHOUSE}

\section{David Chuquer S. ${ }^{1,2 *}$, Darío Torres ${ }^{1}$, Edgar González V. ${ }^{1}$, Jorge Moncayo $^{1}$, Diana Astorga ${ }^{2} \&$ Christian Alcívar $^{1}$}

\author{
Recibido: 19 de marzo 2020 / Aceptado: 8 de junio 2020 \\ Publicado en línea: 16 de junio 2020 \\ DOI: $10.26807 /$ ia.v8i2.136
}

Palabras claves Biogás, desechos de camal, digestión anaerobia, FTIR. Keywords: Biogas, slaughter waste, anaerobic digestion, FTIR.

\section{RESUMEN}

La materia orgánica proveniente de centros de faenamiento, puede aprovecharse mediante digestión anaerobia para producción de biogás y de un fertilizante rico en nutrientes. El monitoreo de la concentración de metano, propano y butano son parámetros relevantes que evidencian la eficiencia de digestión. En el presente estudio, se desarrolló una metodología para la medición de estos

1 Universidad Central del Ecuador, Facultad de Ciencias Químicas, Quito, Ecuador. (*correspondencia: dschuquer@uce.edu.ec; datorresb1@uce.edu.ec; epgonzalez@uce.edu.ec; jemoncayo@uce.edu.ec; cdalcivar@uce.edu.ec)

2 Pontificia Universidad Católica del Ecuador, Facultad de Ciencias Exactas y Naturales, Quito, Ecuador, (dchuquer295@puce.edu.ec; dastorga199@puce.edu.ec) 
hidrocarburos, utilizando espectrofotometría infrarroja de transformadas de Fourier (FTIR) y la asistencia de cálculos químico-cuánticos, que permitieron la asignación de bandas de absorción de cada hidrocarburo en el biogás. Los límites de cuantificación fueron de $0,36 \% \mathrm{~V} / \mathrm{V}$ para propano y butano y 1,47 $\%$ V V para metano. Se realizaron pruebas de biodigestión con desechos del centro de faenamiento municipal del Distrito Metropolitano de Quito, obteniéndose que una mezcla de rumen y estiércol bovino, sometida a $50{ }^{\circ} \mathrm{C}$, generó biogás enriquecido hasta en un $65,4 \% \mathrm{~V} / \mathrm{V}$ de metano posterior a 15 días de biodigestión. Adicionalmente, se produjo digestado sólido y líquido que cumplen con las normativas internacionales para su posible aplicación en el suelo. Los resultados demostraron la potencialidad del método de seguimiento de biodigestión y la posible transformación de desechos de camal en una fuente de energía renovable.

\section{ABSTRACT}

Organic matter from slaughterhouses can be used through anaerobic digestion to produce biogas and a nutrient-rich fertilizer. Monitoring the concentration of methane, propane, and butane are relevant parameters that show the efficiency of digestion. In the present study, a methodology for the measurement of these hydrocarbons was developed, using Fourier transform infrared spectrophotometry (FTIR) and the assistance of chemical-quantum calculations, which allowed the assignment of absorption bands of each hydrocarbon in biogas. The quantification limits were $0.36 \% \mathrm{~V} / \mathrm{V}$ for propane and butane and 1.47 $\% \mathrm{~V} / \mathrm{f}$ for methane. Digestion tests were carried out with waste from the municipal slaughter center of the Metropolitan District of Quito, obtaining that a mixture of rumen and bovine manure, subjected to $50{ }^{\circ} \mathrm{C}$, generated enriched biogas up to $65.4 \% \mathrm{~V} / \mathrm{V}$ after 15 days of digestion. Additionally, solid and liquid digestate produced met with international regulations for possible application in the soil. The results demonstrate the potential of the digestion monitoring method and the possible transformation of litter waste into a renewable energy source. 


\section{INTRODUCCIÓN}

La industria de procesamiento de carne es una de los mayores del mundo (43,7 kg/año per cápita) (FAO, 2018) y utiliza alrededor del $24 \%$ del agua consumida por la industria de alimentos (Bustillo-Lecompte \& Mehrvar, 2015). En Ecuador, hasta el 2018, el ganado bovino, porcino y ovino contabilizo 5,696 millones de cabezas de ganado y 151,683 millones de aves en planteles avícolas (Instituto Nacional de Estadísticas y Censos, 2018). Adicionalmente, en el país se registran 277 mataderos bajo inspección oficial que siguen procedimientos normalizados por la Agencia de Regulación y Control Fito y Zoosanitario (AGROCALIDAD), que basándose en la Resolución DAJ20134B4-0201.0247 buscan el aseguramiento de la calidad de los productos cárnicos (Agrocalidad, 2013, 2018).

Los desechos generados en centros de faenamiento son aguas residuales, material sólido y emisiones gaseosas. Respecto a los desechos sólidos, en base a su origen y carga orgánica, se emplean como mejoradores de suelos favoreciendo la producción orgánica de alimentos (Peñafiel \& Ticona, 2015) y mediante un tratamiento anaerobio la generación de energía sustentable, por lo que su aprovechamiento puede convertir el problema de su disposición final en una oportunidad. Existe una variedad de microorganismos presentes en los desechos de camal los cuales constituyen un riesgo para la salud humana, salud animal y al medio ambiente. En este sentido, trabajadores expuestos a desechos de camal tienen alto riesgo de contaminación con Sallmonella spp, Streptococcus suis, Campylobacter, Leptospirosis, Toxoplasmosis y una gran variedad de virus (Djeffal et al., 2018; Kozdruń et al., 2015; Soares et al., 2015).

Tomando en cuenta la toxicidad de los desechos provenientes de centros de faenamiento, su potencialidad dado su alto contenido en materia orgánica y la necesidad de fuentes de energía renovables debido al agotamiento progresivo de los combustibles fósiles, en las últimas décadas se ha desarrollado las tecnologías de digestión anaerobias para la producción de biogás a partir de estiércol de 
diversos tipos de ganado (Afazeli et al., 2014). El biogás generado está compuesto principalmente por metano $-\mathrm{CH}_{4}(40-70 \%)$ y dióxido de carbono $-\mathrm{CO}_{2}$ (30-50 \%) (Accettola et al., 2008). Hagos (2017) indica que los residuos provenientes de mataderos poseen un alto contenido orgánico y de sólidos volátiles. Los ácidos volátiles de alto peso molecular se convierten durante la fermentación anaerobia en $\mathrm{CH}_{4}, \mathrm{CO}_{2}$ y ácidos orgánicos de cadena corta. Sin embargo, este tipo de desechos posee un alto contenido proteico que por degradación microbiana libera amoniaco, el cual puede inhibir la actividad metanogénica y conducir a una inestabilidad del proceso (Hagos et al., 2017). Para contrarrestar esto se debe regular la proporción $\mathrm{C} / \mathrm{N}$ (30/1) así como los niveles de $\mathrm{pH}$. En este sentido, el seguimiento del proceso es clave para maximizar la obtención de biogás.

Tomando en cuenta estos antecedentes, el estudio presentado tiene como objetivos desarrollar un procedimiento para el seguimiento de un proceso de biodigestión anaerobia (metano- $\mathrm{CH}_{4}$, propano- $\mathrm{C}_{3} \mathrm{H}_{8}$ y butano- $\mathrm{C}_{4} \mathrm{H}_{10}$ ) y evaluar el proceso de biodigestión de desechos provenientes del centro de faenamiento del Distrito Metropolitano de Quito.

\section{MATERIALES Y MÉTODOS}

Se desarrolló una metodología para el análisis de $\mathrm{CH}_{4}, \mathrm{C}_{3} \mathrm{H}_{8}$ y $\mathrm{C}_{4} \mathrm{H}_{10}$ mediante análisis infrarrojo. Para ello se utilizó un espectrofotómetro FTIR Varian 660 con una Short-Path HT Gas Cell marca PIKE Technologies, de $100 \mathrm{~mm}$ de longitud con ventanas de bromuro de potasio de $25 \times 4 \mathrm{~mm}$ de la misma marca. Para la calibración se utilizó dos gases estándares trazables a NIST con las características detalladas en la Tabla 1. Para la dilución de los gases se utilizaron jeringas plásticas de $50 \mathrm{~mL}$ y bolsas Tedlar de 2 L. La limpieza de la celda se realizó con aire ambiente con una bomba de vacío Millipore WP6111580. 
Tabla 1. Gases estándares de calibración

\begin{tabular}{ll}
\hline Rango Alto (RA) & Rango Bajo (RB) \\
\hline Airgas X05NI93CP580000 & Airgas X05NI99CP580002 \\
$30000 \mathrm{~mL} \mathrm{~m}{ }^{-3} \mathrm{CH}_{4}$ & $250 \mathrm{~mL} \mathrm{~m}{ }^{-3} \mathrm{CH}_{4}$ \\
$21000 \mathrm{~mL} \mathrm{~m} \mathrm{C}_{3} \mathrm{H}_{8}$ & $250 \mathrm{~mL} \mathrm{~m} \mathrm{C}_{3} \mathrm{H}_{8}$ \\
$18000 \mathrm{~mL} \mathrm{~m} \mathrm{C}_{4} \mathrm{H}_{10}$ & $250 \mathrm{~mL} \mathrm{~m}^{-3} \mathrm{C}_{4} \mathrm{H}_{10}$ \\
\hline
\end{tabular}

Se estableció el siguiente protocolo de trabajo: se limpió la bolsa Tedlar con aire ambiente por un minuto, utilizando la bomba de vacío. Posterirormente, se homogenizó la bolsa Tedlar con estándar. Luego, se agregó 0,3 L de estándar. Seguido, se preparó las diluciones con la jeringa bajo el esquema establecido en la Tabla 2. Se inyectó $50 \mathrm{~mL}$ de muestra en la celda del FTIR y se finalmente se obtuvo el espectro IR realizando 4 barridos espectrales entre 4000 a 400 $\mathrm{cm}^{-1}$.

El espectro experimental IR del biogás, en fase gaseosa fue correlacionado con espectros vibracionales de $\mathrm{CH}_{4}, \mathrm{C}_{3} \mathrm{H}_{8}$ y $\mathrm{C}_{4} \mathrm{H}_{10}$, determinados por cálculos teóricos químico-cuánticos y generados con el programa Gaussian 09, utilizando el nivel de teoría HF/3-21G con un factor de escalamiento de las frecuencias vibra- cionales calculadas de 0,906 (Irikura et al., 2005). Los espectros calculados se superpusieron, para contrastar la localización y el perfil de bandas de absorción con el espectro experimental (Figura 2), con la finalidad de realizar una asignación tentativa de los principales modos vibracionales.

Con las concentraciones definidas en la Tabla 2, se determinó la precisión inter-día realizando tres repeticiones de las lecturas de cada estándar, en tres días diferentes, ejecutándose en total cuarenta y cinco ensayos. La linealidad se determinó mediante mínimos cuadrados entre absorbancia versus concentración del gas, ajustando una ecuación que coincidió con el origen. Adicionalmente se establecieron los límites de confianza de la curva calculando la desviación estándar de la correlación (Sr) y aplicando la $t$ de student para un $95 \%$ 
de confianza $(1,96)$. Con el mismo Sr, (LD) y cuantificación (LC) (Miller et se calcularon los límites de detección al., 2018).

Tabla 2. Diluciones de estándares

\begin{tabular}{ccccc}
\hline Patrón & Dilución & $\begin{array}{c}\mathrm{CH}_{4} \\
\left(\mathrm{~mL} \mathrm{~m}^{-3}\right)\end{array}$ & $\begin{array}{c}\mathrm{C}_{3} \mathrm{H}_{8} \\
\left(\mathrm{~mL} \mathrm{~m}^{-3}\right)\end{array}$ & $\begin{array}{c}\mathrm{C}_{4} \mathrm{H}_{10} \\
\left(\mathrm{~mL} \mathrm{~m}^{-3}\right)\end{array}$ \\
\hline Aire & 1 & 0 & 0 & 0 \\
RB & 1 & 250 & 250 & 250 \\
RA & 10 & 3000 & 2100 & 1800 \\
RA & 2,5 & 12000 & 8400 & 7200 \\
RA & 1 & 30000 & 21000 & 18000 \\
\hline
\end{tabular}

Para la repetibilidad y reproducibilidad se aplicó un análisis de varianza (ANOVA) de factores totalmente anidados y homogéneos obteniéndose el porcentaje de desviación debida a la repetibilidad y reproducibilidad (Maroto et al., 1999).

Las pruebas de biodigestión de desechos de camal se realizaron bajo el esquema detallado en la Figura 1. Para mantener las muestras a $50{ }^{\circ} \mathrm{C}$, se utilizó un baño térmico Memmert W270.

Se recolectaron muestras compuestas de lodos de planta de tratamiento de aguas residuales (M1), rumen (M2) y una mezcla de rumen y estiércol (M3) en el camal del Distrito Metropolitano de Quito, entidad adscrita a la Empresa Pública Metropolitana de Rastro de Quito. El muestreo se realizó siguiendo la norma NTE INENISO 10381-2 que especifica las técnicas de muestreo de suelo, tomando submuestras en 10 zonas del reservorio que contenía el material y formando una sola muestra compuesta, la cual fue procesada y analizada según el esquema detallado en la Figura 1. 


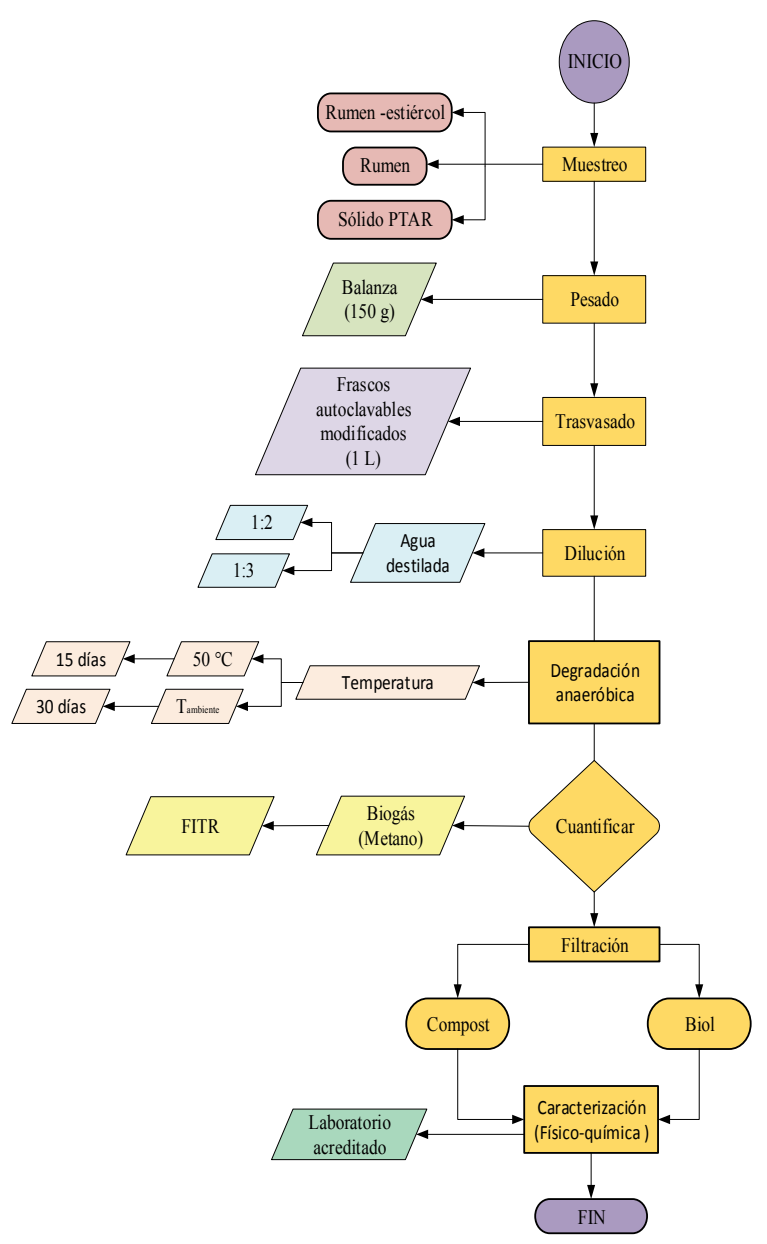

Figura 1. Metodología de digestión anaerobia de muestras

Se realizó un muestreo el 04 de enero de 2019 para determinar las condiciones iniciales del sustrato y se realizó otro muestreo el 08 de agosto de 2019 para evaluar la composición del digestado sólido y líquido. Se agregó agua destilada tipo 2 en pro- porciones 1:2 y 1:3 (desecho:agua). Posteriormente, se realizó la biodigestión anaerobia en frascos autoclavables Boeco, adaptando un tapón de caucho en la tapa original para recolectar el biogás. 


\section{RESULTADOS}

Las asignaciones realizadas con la asistencia de cálculos químico-cuánticos, permitieron establecer un método de cuantificación (Comeford \& Gould, 1961; Pele et al., 2011). Las bandas de absorción experimentales en el IR asignadas con la asistencia de cálculos teóricos fueron $1303 \mathrm{~cm}^{-1}$ para $\mathrm{CH}_{4}$ y $2964 \mathrm{~cm}^{-1}$ para $\mathrm{C}_{3} \mathrm{H}_{8^{-}}$
$\mathrm{C}_{4} \mathrm{H}_{10}$, con la finalidad de monitorizar la producción de dichos gases durante la biodigestión anaerobia (Figura 2). Las curvas de calibración de absorbancia versus concentración de los gases a ambos números de ondas $(v)$ presentaron relaciones lineales, cuyos parámetros se resumen en la Tabla 3.

Tabla 3. Parámetros de linealidad

\begin{tabular}{lll}
\hline Parámetro & $\mathrm{CH}_{\mathbf{4}}$ & $\mathrm{C}_{\mathbf{3}} \mathrm{H}_{\mathbf{8}}-\mathrm{C}_{\mathbf{4}} \mathrm{H}_{10}$ \\
\hline$v\left(\mathrm{~cm}^{-1}\right)$ & 1303 & 2964 \\
Recta & $\mathrm{A}=6,7711 \times 10^{-6 *} \mathrm{C}_{\mathrm{m}}$ & $\mathrm{A}=3,0566 \times 10^{-5 *} \mathrm{C}_{\mathrm{pb}}$ \\
$\mathrm{R}^{2}$ & 0,9921 & 0,9997 \\
$\left.\mathrm{LD}(\mathrm{mL} \mathrm{m})^{-3}\right)$ & 4399 & 1065 \\
$\mathrm{LC}\left(\mathrm{mL} \mathrm{m} \mathrm{m}^{-3}\right)$ & 14665 & 3551 \\
\hline
\end{tabular}

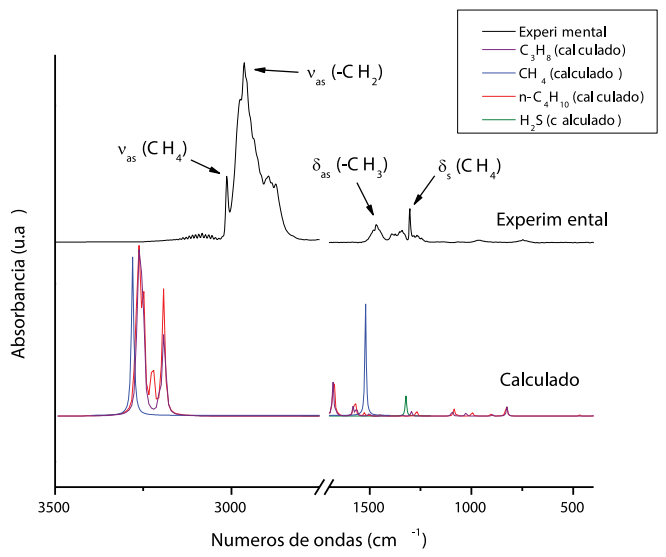

Figura 2. Espectro FTIR experimental en fase de gas y calculado 
En las tablas 4 y 5 se reportan los resultados del análisis de repetibilidad y reproducibilidad aplicando el ANOVA. Se indica el porcentaje de variación debido a la repetibilidad (\%CVr) y reproducibilidad (CVR), así como la F de Fischer con un $95 \%$ de confianza y el coeficiente de Horwitz (\%CVh) para cada nivel de concentración (Miller et al., 2018). El criterio de aceptabilidad para la repetibilidad fue $0,5 \%$ CVh $>\% C V r$ y para la reproducibilidad $0,67 * \% \mathrm{CVh}>\% \mathrm{CVR}$.

\section{Tabla 4. Repetitibilidad y reproducibilidad para la calibración de $\mathrm{CH}_{4}$}

\begin{tabular}{ccccc}
\hline $\mathrm{CH}_{\mathbf{4}}\left(\mathrm{mL} \mathrm{m}^{-3}\right)$ & $\mathrm{CVr}(\%)$ & CVR (\%) & $\mathrm{F}_{\text {calculado }}{ }^{*}$ & $\mathrm{CVh}(\%)$ \\
\hline 250 & 19,7 & 48,2 & 16,0 & 7,0 \\
3000 & 6,8 & 11,3 & 6,4 & 4,8 \\
12000 & 1,0 & 8,9 & 234,0 & 3,9 \\
30000 & 0,3 & 7,3 & 1501,0 & 3,4 \\
\hline
\end{tabular}

${ }^{*} \mathrm{~F}_{\text {crítico }}=5,143$ para 3 repeticiones de cada estándar en tres días diferentes

Tabla 5. Repetibilidad y reproducibilidad para la calibración de $\mathrm{C}_{3} \mathrm{H}_{8}-\mathrm{C}_{4} \mathrm{H}_{10}$

\begin{tabular}{ccccc}
\hline $\mathrm{C}_{\mathbf{3}} \mathrm{H}_{\mathbf{8}}-\mathrm{C}_{\mathbf{4}} \mathrm{H}_{\mathbf{1 0}}\left(\mathrm{mL} \mathrm{m}^{-3}\right)$ & $\mathrm{CVr}(\%)$ & $\mathrm{CVR}(\%)$ & $\mathrm{F}_{\text {calculado }}{ }^{*}$ & $\mathrm{CVh}(\%)$ \\
\hline 500 & 3,1 & 9,2 & 24,5 & 6,3 \\
3900 & 5,3 & 4,9 & 0,5 & 4,6 \\
15600 & 0,9 & 1,4 & 5,5 & 3,7 \\
39000 & 0,3 & 0,3 & 0,6 & 3,3 \\
\hline
\end{tabular}

*Fcrítico $=5,143$ para 3 repeticiones de cada estándar en tres días diferentes

Posteriormente, se realizó la digestión anaerobia de tres muestras de desechos de camal: M1, M2 y M3. La Figura 3 muestra la producción de
$\mathrm{CH}_{4}$ durante 24 días a temperatura ambiente y a $50{ }^{\circ} \mathrm{C}$, con una relación desechos : agua de 1:2 y 1:3. 

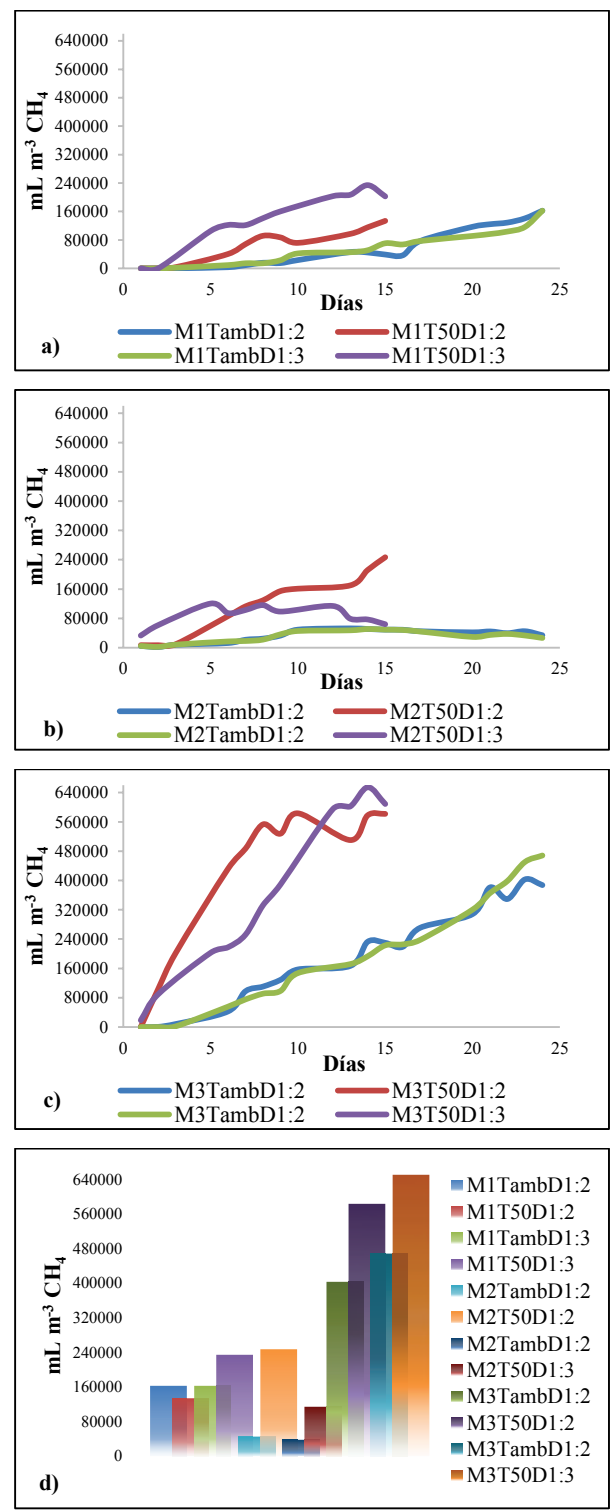

Figura 3. Concentración de $\mathrm{CH}_{4}$ durante biodigestión para a) $\mathrm{M1}$, b) $\mathrm{M2}$, c) $\mathrm{M} 3$ y d) $\mathrm{CH}_{4}$ máximo de cada serie de experimentos 
La caracterización fisicoquímica y microbiológica del biol y digestado fue realizada en un laboratorio acreditado (ALS-global) y se detallan en la Tabla 6.

El análisis estadístico para determinar la influencia de las variables en la biodigestión fue realizado en Stat- graphics ${ }^{\circledR}$ mediante un diseño factorial $3 \times 2 \times 2$ y se muestra en la Tabla 7. Existió un efecto estadístico significativo entre las variables de diseño en la concentración de $\mathrm{CH}_{4}$ (función respuesta) cuando el parámetro $\mathrm{p}<$ 0,05 para un nivel de confianza del $95 \%$.

Tabla 6. Composición de sustrato, biol y digestado sólido

\begin{tabular}{|c|c|c|c|c|c|c|c|c|}
\hline Parámetros & Unidades & Método & M1 & M2 & M3 & $\begin{array}{l}\text { Digestado } \\
\text { M3T50d1:3 }\end{array}$ & Unidades & $\begin{array}{c}\text { Biol } \\
\text { M3T50d1:3 }\end{array}$ \\
\hline $\begin{array}{l}\text { Nitrógeno } \\
\text { Total } \\
\text { Kjendahl }\end{array}$ & $\mathrm{mg} \mathrm{kg}^{-1}$ & $\begin{array}{c}\text { SM 4500- } \\
\text { Norg Cl }\end{array}$ & 13691,3 & 17383,7 & 17381,2 & 10300,2 & $\mathrm{mg} \mathrm{L}^{-1}$ & 371 \\
\hline Potasio & $\mathrm{mg} \mathrm{kg}^{-1}$ & $\begin{array}{c}\text { USEPA } \\
3050 \mathrm{~B} \\
7610 \\
\end{array}$ & 4022,6 & 4776 & 11122 & 1532,3 & $\mathrm{mg} \mathrm{L}^{-1}$ & 195 \\
\hline $\begin{array}{l}\text { Carbono } \\
\text { orgánico }\end{array}$ & $\%$ & $\begin{array}{c}\text { WALKLEY } \\
\& \text { BLACK, } \\
1934\end{array}$ & 25,37 & 36,65 & 30,85 & 35,38 & - & - \\
\hline Calcio & $\mathrm{mg} \mathrm{kg}^{-1}$ & $\begin{array}{c}\text { USEPA } \\
3010 \mathrm{~A} \\
3050 \mathrm{~B}\end{array}$ & 15515,8 & 2149,2 & 2669,3 & 8427,8 & $\mathrm{mg} \mathrm{L}^{-1}$ & 225 \\
\hline Fósforo & $\mathrm{mg} \mathrm{kg}^{-1}$ & $\begin{array}{c}\text { SM 4500-P } \\
\text { B y } 4500-P \\
\text { C }\end{array}$ & 621,7 & 201,5 & 576,7 & 1214,9 & $\mathrm{mg} \mathrm{L}^{-1}$ & 160,75 \\
\hline Magnesio & $\mathrm{mg} \mathrm{kg}^{-1}$ & $\begin{array}{l}\text { USEPA } \\
3010 \mathrm{~A} \\
3050 \mathrm{~B}\end{array}$ & 637,6 & 529,2 & 567,2 & 395,3 & $\mathrm{mg} \mathrm{L}^{-1}$ & 17,66 \\
\hline $\begin{array}{l}\text { Potencial de } \\
\text { Hidrógeno }\end{array}$ & $\mathrm{UpH}$ & $\begin{array}{l}\text { USEPA } \\
9045 \mathrm{D}\end{array}$ & 8,29 & 7,69 & 7,48 & 7,16 & $\mathrm{mg} \mathrm{L}^{-1}$ & 7,24 \\
\hline $\begin{array}{l}\text { Sólidos } \\
\text { Totales }\end{array}$ & $\%$ & $\begin{array}{l}\text { USEPA } \\
160.3 \\
\end{array}$ & 23,66 & 12,25 & 12,57 & 10,28 & $\mathrm{mg} \mathrm{L}^{-1}$ & 8306 \\
\hline Sulfatos & $\mathrm{mg} \mathrm{kg}^{-1}$ & $\begin{array}{c}\text { USEPA } \\
375.4 \mathrm{SO} 4\end{array}$ & 77,4 & 58 & 91,6 & $<50$ & $\mathrm{mg} \mathrm{L}^{-1}$ & 171 \\
\hline $\begin{array}{c}\text { Sólidos } \\
\text { Suspendidos } \\
\text { Totales }\end{array}$ & $\%$ & $\begin{array}{l}\text { USEPA } \\
160.3\end{array}$ & 23,35 & 12,2 & 12,49 & - & - & - \\
\hline
\end{tabular}


InfoANALÍTICA 8(2)

Julio 2020

\begin{tabular}{|c|c|c|c|c|c|c|c|c|}
\hline $\begin{array}{l}\text { Demanda } \\
\text { Química de } \\
\text { Oxigeno }\end{array}$ & $\mathrm{mg} \mathrm{kg}^{-1}$ & $\begin{array}{c}\text { WALKLEY } \\
\& \text { BLACK } \\
1934\end{array}$ & 253700 & 366500 & 308500 & - & - & - \\
\hline $\begin{array}{c}\text { Sólidos } \\
\text { Disueltos } \\
\text { Totales }\end{array}$ & $\%$ & $\begin{array}{r}\text { USEPA } \\
160.3\end{array}$ & 0,31 & 0,05 & 0,08 & - & - & - \\
\hline Humedad & $\%$ & $\begin{array}{c}\text { NMX-AA- } \\
1984\end{array}$ & $16-77,27$ & 87,43 & 90,92 & 89,72 & - & - \\
\hline $\begin{array}{l}\text { Sólidos } \\
\text { Secos }\end{array}$ & $\%$ & $\begin{array}{c}\text { NMX-AA- } \\
16-1984\end{array}$ & 22,73 & 12,57 & 9,08 & 10,28 & - & - \\
\hline Cobre & $\mathrm{mg} \mathrm{kg}^{-1}$ & $\begin{array}{c}\text { USEPA } \\
3010 \mathrm{~A} \\
3050 \mathrm{~B} \\
\text { EPA } 7210\end{array}$ & 5,1 & $<5,0$ & 5,8 & 26,8 & $\mathrm{mg} \mathrm{L}^{-1}$ & 0,24 \\
\hline Níquel & $\mathrm{mg} \mathrm{kg}^{-1}$ & $\begin{array}{c}\text { USEPA } \\
3010 \mathrm{~A} \\
3050 \mathrm{~B}\end{array}$ & 6,9 & 7,2 & 6,2 & 9,2 & $\mathrm{mg} \mathrm{L}^{-1}$ & 0,06 \\
\hline Arsénico & $\mathrm{mg} \mathrm{kg}^{-1}$ & SM $3114 \mathrm{~B}$ & 0,3 & 0,3 & 0,29 & $<0,10$ & $\mu \mathrm{g} \mathrm{L}^{-1}$ & $<2,00$ \\
\hline Bario & $\mathrm{mg} \mathrm{kg}^{-1}$ & $\begin{array}{c}\text { USEPA } \\
3010 \mathrm{~A} \\
3050 \mathrm{~B}\end{array}$ & 36,8 & 54 & 46,3 & 53,2 & $\mathrm{mg} \mathrm{L}^{-1}$ & 0,99 \\
\hline Cadmio & $\mathrm{mg} \mathrm{kg}^{-1}$ & 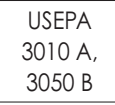 & 2,76 & 2,29 & 2,14 & 1,53 & $\mathrm{mg} \mathrm{L}^{-1}$ & $<0,02$ \\
\hline Mercurio & $\mathrm{mg} \mathrm{kg}^{-1}$ & $\begin{array}{l}\text { USEPA } \\
7471 \mathrm{~B} \\
\end{array}$ & 0,1 & $<0,1$ & 0,1 & $<0,10$ & $\mu g \mathrm{~L}^{-1}$ & $<2,0$ \\
\hline Plata & $\mathrm{mg} \mathrm{kg}^{-1}$ & $\begin{array}{l}\text { USEPA } \\
3050 \mathrm{~B}, \\
7760 \mathrm{~A} \\
\end{array}$ & 2,5 & $<2,5$ & 2,5 & $<2,5$ & $\mathrm{mg} \mathrm{L}^{-1}$ & 0,07 \\
\hline Plomo & $\mathrm{mg} \mathrm{kg}^{-1}$ & $\begin{array}{l}\text { USEPA } \\
3010 \mathrm{~A}, \\
3050 \mathrm{~B}\end{array}$ & 12,4 & 13,9 & 12,9 & 13,8 & $\mathrm{mg} \mathrm{L}^{-1}$ & 0,17 \\
\hline Zinc & $\mathrm{mg} \mathrm{kg}^{-1}$ & $\begin{array}{c}\text { USEPA } \\
3010 \mathrm{~A}, \\
3050 \mathrm{~B}\end{array}$ & 9,7 & 23,4 & 7,1 & 44,1 & $\mathrm{mg} \mathrm{L}^{-1}$ & 1,1 \\
\hline Cromo Total & $\mathrm{mg} \mathrm{kg}^{-1}$ & 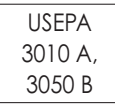 & 12 & 14,8 & 14,7 & 3,8 & $\mathrm{mg} \mathrm{L}^{-1}$ & 0,09 \\
\hline Molibdeno & $\mathrm{mg} \mathrm{kg}^{-1}$ & $\begin{array}{c}\text { USEPA } \\
3010 \mathrm{~A} \\
3050 \mathrm{~B}\end{array}$ & 12,5 & $<12,5$ & 12,5 & $<12,5$ & $\mathrm{mg} \mathrm{L}^{-1}$ & $<0,25$ \\
\hline Selenio & $\mathrm{mg} \mathrm{kg}^{-1}$ & $\begin{array}{c}\text { SM } 3120 B \\
\text { USEPA } \\
3050 \text { B }\end{array}$ & 0,1 & $<0,100$ & 0,1 & $<0,1$ & $\mathrm{mg} \mathrm{L}^{-1}$ & $<0,010$ \\
\hline $\begin{array}{c}\text { Coliformes } \\
\text { Fecales }\end{array}$ & NMP $g^{-1}$ & $\begin{array}{c}\text { SM } 9221 \mathrm{~B}, \\
\mathrm{E}, \mathrm{F}\end{array}$ & 1400 & 7000 & 7000 & 0 & $\mathrm{NMP} / 100 \mathrm{~mL}$ & 2 \\
\hline $\begin{array}{c}\text { Coliformes } \\
\text { Totales }\end{array}$ & NMP g ${ }^{-1}$ & $\begin{array}{c}\text { SM } 9221 \mathrm{~B}, \\
\mathrm{E}, \mathrm{F}\end{array}$ & 2800 & 210000 & 93000 & 0 & $\mathrm{NMP} / 100 \mathrm{~mL}$ & 13 \\
\hline
\end{tabular}




\begin{tabular}{lcccccccc}
\hline $\begin{array}{c}\text { Huevos de } \\
\text { Helmintos }\end{array}$ & Huevos g-1 & $\begin{array}{c}\text { NMX-AA- } \\
113-S C F- \\
2012\end{array}$ & 1 & $<1$ & 1 & $<1$ & Huevos $\mathrm{mL}^{-1}$ & 0 \\
\hline Salmonella & UFC g $^{-1}$ & $\begin{array}{c}3 \mathrm{M} \\
\text { PETRIFIM }{ }^{\circledR}\end{array}$ & 0 & 0 & 0 & 0 & UFC $\mathrm{mL}^{-1}$ & 0 \\
\hline
\end{tabular}

SM= Standard Methods, USEPA= United States Environmental Protection Agency, NMX=Norma Mexicana

Tabla 7. ANOVA para $\mathrm{CH}_{4}$

\begin{tabular}{lcc}
\hline Parámetros & Razón -F & Valor-p \\
\hline A: Desecho & 20845,71 & 0,00 \\
B: Temperatura & 10339,58 & 0,00 \\
C: Dilución & 6,67 & 0,02 \\
AB & 1963,84 & 0,00 \\
AC & 342,21 & 0,00 \\
BC & 2,98 & 0,11 \\
ABC & 386,36 & 0,00 \\
\hline
\end{tabular}

\section{DISCUSIÓN}

La espectroscopia vibracional IR brinda la ventaja de ser una técnica no destructiva, de rápida y fácil utilización. La ventaja de cuantificar varios gases como $\mathrm{CH}_{4}, \mathrm{CO}_{2}, \mathrm{C}_{4} \mathrm{H}_{10}$ y $\mathrm{C}_{3} \mathrm{H}_{8}$ en mezclas complejas de gases (Hepburn et al., 2015), promovió su utilización, la cual se fundamenta en la identificación de los modos de vibración característicos de cada molécula y se asiste en la asignación por cálculos teóricos químico-cuánticos. La Tabla 3 muestra la proporcionalidad de las relaciones absorbancia versus concentración de $\mathrm{CH}_{4}$ y de
$\mathrm{C}_{3} \mathrm{H}_{8}-\mathrm{C}_{4} \mathrm{H}_{10}$ con coeficientes de correlación $\left(\mathrm{R}^{2}\right)$ cercanos a 1 .

En base a los resultados obtenidos, el rango lineal de mediciones directas de biogás se sitúa entre los $14665 \mathrm{~mL}$ $\mathrm{m}^{-3}(1,47 \% \mathrm{~V} / \mathrm{V})$ y $30000 \mathrm{~mL} \mathrm{~m} \mathrm{~m}^{-3}$ $(3,00 \% \mathrm{~V} / \mathrm{V}) \mathrm{de} \mathrm{CH}_{4}$ y entre los 3551 $\mathrm{mL} \mathrm{m}{ }^{-3}(0,36 \% \mathrm{~V} / \mathrm{V})$ y $40000 \mathrm{~mL} \mathrm{~m}^{-3}$ $\left(4,00 \%\right.$ V/V) de $\mathrm{C}_{3} \mathrm{H}_{8}-\mathrm{C}_{4} \mathrm{H}_{10}$. Las diluciones de las muestras de biogás (hasta FD=25) han permitido evaluar concentraciones de hasta el 65,4\% V/N de $\mathrm{CH}_{4}$. 
Los límites de detección y cuantificación obtenidos en este trabajo, permitirán aplicar este procedimiento en la determinación de concentraciones de $\mathrm{CH}_{4}$ en biodigestores donde las proporciones de estos gases superan el 1,5\%V/V incluso en las primeras etapas de la biodigestión anaerobia de residuos sólidos (Deublein \& Steinhauser, 2008). Si bien es cierto, existen sensores electroquímicos con límites de detección más bajos (entre 16 y $170 \mathrm{~mL} \mathrm{~m}^{-3}$ ) (Honeycutt et al., 2019), la saturación de estos sensores se da a concentraciones superiores al $5 \% \mathrm{~V} / \mathrm{V}$ de $\mathrm{CH}_{4}$, por lo cual se usan principalmente en la detección de explosividad y dosis letal en ambiente cerrados. Los métodos de análisis más usados para la cuantificación de $\mathrm{CH}_{4}$ en concentraciones encima del $5 \%$ V $\mathrm{V}$ son los métodos de infrarrojo no dispersivo (NDIR) y las cámaras infrarrojas (Reinelt et al., 2017). Respecto a la precisión del método, es necesaria su calibración cada vez que se vaya a realizar los análisis. El método desarrollado en este trabajo presenta una precisión para $\mathrm{CH}_{4}$ descrita en la Tabla 4, que es similar en el rango lineal a la de los sensores NDIR (entre el 7,3 y 19,8 \%) (Pham et al., 2013).
La Figura 3 muestra las curvas de generación de biogás para $M 1, M 2$ y M3, así como los valores máximos de $\mathrm{CH}_{4}$ generados en la biodigestión. El tratamiento M3T50D1:3 (Mezcla rumen-estiércol a $50{ }^{\circ} \mathrm{C}$ y dilución 1:3) es la opción que genera un biogás más enriquecido en $\mathrm{CH}_{4}(65,4 \%)$ después de 15 días de digestión acorde a los procesos termofílicos (Lagrange, 1979). Es remarcable que el aumento de temperatura en el proceso provoca cambios sustanciales en la velocidad de generación del $\mathrm{CH}_{4}$, mostrando resultados similares a otros estudios reportados (Accettola et al., 2008; Varnero-Moreno, 2011).

La Tabla 7 muestra los resultados del diseño experimental aplicado, teniendo la concentración de $\mathrm{CH}_{4}$ como función respuesta y tomando como factores de proceso el tipo de desecho (M1, M2 y M3), la temperatura (ambiente y $50^{\circ} \mathrm{C}$ ) y la relación desecho:agua (1:2 y 1:3). Se evidenció que todas las variables influyen significativamente en la producción de $\mathrm{CH}_{4}$. Adicionalmente, la prueba de rangos múltiples mostro que la generación de $\mathrm{CH}_{4}$ con M1 y M2 no tiene diferencias significativas, por lo que el estiércol en la mezcla con el 
rumen favorece la producción de un biogás más enriquecido en $\mathrm{CH}_{4}$. Los resultados mostraron que la temperatura es la variable que aporta la mayor influencia en la producción de $\mathrm{CH}_{4}$. Las bacterias metanogénicas prefieren un medio con temperaturas mayores a $30{ }^{\circ} \mathrm{C}$. Valores debajo de los $15{ }^{\circ} \mathrm{C}$ disminuyen la producción de $\mathrm{CH}_{4}$ por reducción de la población metanogénica y aumenta la disolución de gases en el agua. A temperaturas mayores a $15{ }^{\circ} \mathrm{C}$, la solubilidad de los gases disminuye, siendo un efecto positivo para los microorganismos ya que disminuye el riesgo a ser expuestos a gases tóxicos. Sin embargo, gases como el $\mathrm{CO}_{2}$ que proporciona alcalinidad al medio también disminuye, lo que provoca un aumento del $\mathrm{pH}$ generando efectos de inhibición en ambientes con altas concentraciones de amoníaco. Los microorganismos necesitan que el $\mathrm{pH}$ y la temperatura sean controlados y constantes en cada etapa metabólica, tal es el caso que, si existen cambios de temperatura de $\pm 0,5{ }^{\circ} \mathrm{C}$, el proceso de biodigestión se desestabiliza (Tezel et al., 2011).

Si bien la dilución del desecho afecta al proceso, está variable puede ser excluida dependiendo del tipo de desecho como lo detallan los resultados óptimos de concentración de $\mathrm{CH}_{4}$ en M1 y M2. La actividad anaerobia opera en mejores condiciones con un nivel de sólidos totales entre el 8 y $12 \%$ en digestores semicontínuos y entre un 40 y $60 \%$ en digestores discontínuos (MINENERGIA et al., 2011).

Finalmente, en la Tabla 6 se evidencia que las muestras de desechos de camal reunieron las condiciones adecuadas de $\mathrm{pH}$, sólidos totales y la relación C/N (30/1), para realizar el proceso de degradación anaerobia, obteniendo resultados favorables en la producción de biogás. La literatura reporta que el uso de biol y digestado sólido obtenido por digestión anaerobia mejora la calidad de los suelos debido al aumento de biomasa microbiológica y la introducción de fosforo y nitrógeno así como la reducción de la densidad del suelo y el aumento de la conductividad hidráulica (Nkoa, 2014).

El contenido de macronutrientes en el digestado sólido, obtenido de la muestra M3T50D1:3, fue comparado con estándares alemanes sobre la 
composición de fertilizantes orgánicos (al menos de 0,5\% de N, 0,3\% de $\mathrm{P}$ y $0,4 \%$ de $\mathrm{K}$ ), mostrando que el digestado sólido requiere la adición de fertilizantes minerales para ejercer una influencia significativa en el suelo. El nivel de metales pesados en el digestado sólido y el biol cumplen con los límites permisibles de biosólidos aplicables como acondicionantes de suelo expuestos en normativas norteamericanas (CFR-US, 1993). Respecto a los parámetros microbiológicos se logró una reducción del
99,9 \% de coliformes fecales y totales en el biol y digestado sólido de la muestra M3T50D1:3, por lo que la biodigestión termofílica tiene un efecto significativo en la disminución de patógenos.

Con estos resultados se puede decir que tanto el biol como el digestado sólido pueden ayudar a mejorar las características fisicoquímicas de los suelos y disminuir el uso de fertilizantes sintéticos

\section{CONCLUSIÓN}

El método desarrollado, mediante espectroscopia FTIR, para la cuantificación de $\mathrm{CH}_{4}$ y $\mathrm{C}_{3} \mathrm{H}_{8}-\mathrm{C}_{4} \mathrm{H}_{10}$ en muestras de biogás es satisfactorio, sin embargo, su utilización está restringida a la calibración cada vez que se vaya a ejecutar la determinación experimental.

El proceso de digestión anaerobia de desechos del camal del DMQ tiene la potencialidad de generar biogás. El tipo de desecho y la temperatura son factores fundamentales que mejoran el rendimiento, obteniéndose que una mezcla de rumen-estiércol bo- vino, sometida a una temperatura de $50{ }^{\circ} \mathrm{C}$ pueda generar biogás enriquecido hasta en un $65,4 \% \mathrm{~V} / \mathrm{V}$ posterior a 15 días del inicio de la biodigestión.

Mediante el análisis fisicoquímico y microbiológico del digestado sólido y biol obtenido, se determinó que estos productos cumplen con los lineamientos relacionados a biosólidos para su posible aplicación como acondicionantes de suelo y fertilizantes con la ayuda de otros fertilizantes minerales. 


\section{AGRADECIMIENTO}

Los autores agradecen el financia- yecto No. 24 "Convocatoria 2016". miento de la U.C.E. mediante el pro-

\section{LISTA DE REFERENCIAS}

Accettola, F., Guebitz, G. M., \& Schoeftner, R. (2008). Siloxane removal from biogas by biofiltration: biodegradation studies. Clean Technologies and Environmental Policy, 10(2), 211-218. https://doi.org/10.1007/s10098-007-0141-4

Afazeli, H., Jafari, A., Rafiee, S., \& Nosrati, M. (2014). An investigation of biogas production potential from livestock and slaughterhouse wastes. Renewable and Sustainable Energy Reviews, 34, 380-386. https://doi.org/10.1016/j.rser.2014.03.016

Agrocalidad. (2013). Manual de procedimientos para la inspeccion y habilitacion de mataderos (Resolución DAJ-20134B4-0201.0247). http://www.agrocalidad.gob. ec/wp-content/uploads/2015/07/Manual-procedimiento-inspeccion-y-habilitacion-Mataderos-DAJ-20134B4-0201.0247.pdf

Agrocalidad. (2018). Listado de mataderos bajo inspección oficial - MABIO. http://www. agrocalidad.gob.ec/documentos/inocuidad/listado-de-mataderos-mabio-a-abril2018.pdf

Bustillo-Lecompte, C. F., \& Mehrvar, M. (2015). Slaughterhouse wastewater characteristics, treatment, and management in the meat processing industry: A review on trends and advances. Journal of Environmental Management, 161, 287-302. https://doi.org/10.1016/j.jenvman.2015.07.008

CFR-US. (1993). Standards for the use or disponsal of sewage sludge. https://www. law.cornell.edu/cfr/text/40/part-503 
Comeford, J. J., \& Gould, J. H. (1961). Infrared spectra of solid hydrocarbons at very low temperatures. Journal of Molecular Spectroscopy, 5(1-6), 474-481. https://doi.org/10.1016/0022-2852(61)90110-2

Djeffal, S., Mamache, B., Elgroud, R., Hireche, S., \& Bouaziz, O. (2018). Prevalence and risk factors for Salmonella spp. contamination in broiler chicken farms and slaughterhouses in the northeast of Algeria. Veterinary World, 11(8), 1102-1108. https://doi.org/10.14202/vetworld.2018.1102-1108

FAO. (2018). Food Outlook. Biannual Report on Global Food Markets. http://www.fao. org/3/ca2320en/CA2320EN.pdf

Hagos, K., Zong, J., Li, D., Liu, C., \& Lu, X. (2017). Anaerobic co-digestion process for biogas production: Progress, challenges and perspectives. Renewable and Sustainable Energy Reviews, 76, 1485-1496. https://doi.org/10.1016/j.rser.2016.11.184

Hepburn, C. A., Vale, P., Brown, A. S., Simms, N. J., \& McAdam, E. J. (2015). Development of on-line FTIR spectroscopy for siloxane detection in biogas to enhance carbon contactor management. Talanta, 141, 128-136. https://doi.org/10.1016/ j.talanta.2015.03.063

Honeycutt, W. T., Ley, M. T., \& Materer, N. F. (2019). Precision and Limits of Detection for Selected Commercially Available, Low-Cost Carbon Dioxide and Methane Gas Sensors. Sensors, 19(14), 3157. https://doi.org/10.3390/s19143157

Instituto Nacional de Estadísticas y Censos. (2018). Estadísticas Agropecuarias. ESPAC 2018. http://www.ecuadorencifras.gob.ec/estadisticas-agropecuarias-2/

Irikura, K. K., Johnson, R. D., \& Kacker, R. N. (2005). Uncertainties in Scaling Factors for ab Initio Vibrational Frequencies. The Journal of Physical Chemistry A, 109(37), 8430-8437. https://doi.org/10.1021/jp052793n

Kozdrun, W., Czekaj, H., \& Sty , N. (2015). Avian zoonoses - a review. Bulletin of the Veterinary Institute in Pulawy, 59(2), 171-178. https://doi.org/10.1515/bvip-20150026

Lagrange, B. (1979). Biomethane. Principes, Techniques, Utilisation. Vol.2 (Edisud (ed.)). 
Maroto, A., Riu, J., Boqué, R., \& Xavier Rius, F. (1999). Estimating uncertainties of analytical results using information from the validation process. Analytica Chimica Acta, 391(2), 173-185. https://doi.org/10.1016/S0003-2670(99)00111-7

Miller, J., Miller, J., \& Miller, L. (2018). Statistics and Chemometrics for Analytical Chemistry (7th ed.). Harlow: Pearson Education Limited.

MINENERGIA, PNUD, FAO, \& GEF. (2011). Manual de Biogás. http://www.fao.org/3 /as400s/as400s.pdf

Nkoa, R. (2014). Agricultural benefits and environmental risks of soil fertilization with anaerobic digestates: a review. Agronomy for Sustainable Development, 34(2), 473-492. https://doi.org/10.1007/s13593-013-0196-z

Pele, L., Šebek, J., Potma, E. O., \& Benny Gerber, R. (2011). Raman and IR spectra of butane: Anharmonic calculations and interpretation of room temperature spectra. Chemical Physics Letters, 515(1-3), 7-12. https://doi.org/10.1016/j.cplett.2011 .09 .015

Peñafiel, W., \& Ticona, D. (2015). Ruminal De Bovino - Matadero Municipal De La Paz. Revista de Investigación e Innovación Agropecuaria y de Recursos Naturales, 8790.

Pham, C. H., Triolo, J. M., Cu, T. T. T., Pedersen, L., \& Sommer, S. G. (2013). Validation and Recommendation of Methods to Measure Biogas Production Potential of Animal Manure. Asian-Australasian Journal of Animal Sciences, 26(6), 864-873. https://doi.org/10.5713/ajas.2012.12623

Reinelt, T., Delre, A., Westerkamp, T., Holmgren, M. A., Liebetrau, J., \& Scheutz, C. (2017). Comparative use of different emission measurement approaches to determine methane emissions from a biogas plant. Waste Management, 68, 173-185. https://doi.org/10.1016/j.wasman.2017.05.053

Soares, T. C. S., Gottschalk, M., Lacouture, S., Megid, J., Ribolla, P. E. M., Pantoja, J. C. de F., \& Paes, A. C. (2015). Streptococcus suis in employees and the environment of swine slaughterhouses in São Paulo, Brazil: Occurrence, risk factors, serotype distribution, and antimicrobial susceptibility. Canadian Journal of Veterinary Re- 
search = Revue Canadienne de Recherche Veterinaire, 79(4), 279-284. http://www.ncbi.nlm.nih.gov/pubmed/26424907

Tezel, U., Tandukar, M., \& Pavlostathis, S. G. (2011). Anaerobic Biotreatment of Municipal Sewage Sludge. In Comprehensive Biotechnology (pp. 447-461). Elsevier. https://doi.org/10.1016/B978-0-08-088504-9.00329-9

Varnero-Moreno, M. T. (2011). Manual de Biogás. http://www.fao.org/3/as400s/as 400s.pdf

Walkley, A., \& Black, I. A. (1934). An Examination of the Degtjareff Method for Determining Soil Organic Matter, and a Proposed Modification of the Chromic Acid Titration Method. Soil Science, 37(1), 29-38. https://doi.org/10.1097/00010694193401000-00003 